\title{
Genomic analysis of a rare human tumor
}

\author{
Steven JM Jones ${ }^{1 *}$, Janessa Laskin², Yvonne Y Li', Obi L Griffith, Jianghong An¹, Mikhail Bilenky', \\ Yaron S Butterfield', Timothee Cezard ${ }^{1}$, Eric Chuah', Richard Corbett', Anthony Fejes', Malachi Griffith', John Yee ${ }^{3}$, \\ Montgomery Martin², Michael Mayo ${ }^{1}$, Nataliya Melnyk ${ }^{4}$, Ryan D Morin', Trevor J Pugh', Tesa Severson ${ }^{1}$, \\ Sohrab P Shah ${ }^{4,5}$, Margaret Sutcliffe ${ }^{2}$, Angela Tam¹, Jefferson Terry ${ }^{4}$, Nina Thiessen 1, Thomas Thomson², \\ Richard Varhol ${ }^{1}$, Thomas Zeng ${ }^{1}$, Yongjun Zhao ${ }^{1}$, Richard A Moore ${ }^{1}$, David G Huntsman³ ${ }^{3}$ Inanc Birol ${ }^{1}$, Martin Hirst ${ }^{1}$, \\ Robert A Holt ${ }^{1}$, Marco A Marra ${ }^{1}$
}

From UT-ORNL-KBRIN Bioinformatics Summit 2010

Cadiz, KY, USA. 19-21 March 2010

\section{Background}

The introduction of next-generation DNA sequencing devices into the field of oncology provides an unprecedented mechanism to determine the underlying genetic changes that have occurred within a tumor and also the changes that accrue during treatment. An enhanced understanding of the oncogenic mechanisms could have an immediate clinical role in the treatment of rare tumors - where treatment protocols do not exist and their rarity would indicate that clinical trials would be unlikely to be undertaken for their establishment.

\section{Results}

We have investigated the utility of massively parallel sequencing to characterize a rare adenocarcinoma of the tongue, before and after treatment. In the pre-treatment tumor we identified 7,629 genes within regions of copy number gain, 1,078 genes exhibited increased expression relative to the blood and unrelated tumors and four genes contained somatic protein-coding mutations. Our analysis suggested the tumor cells were driven by the RET oncogene and its other pathway constituents. Genes whose protein products are targeted by the RET inhibitors sunitinib and sorafenib correlated with being amplified and or highly expressed. Consistent with our observations subsequent administration of sunitinib was associated with stable disease lasting 4 months, after which the lung lesions began to grow. Administration of sorafenib and sulindac provided disease stabilization for an additional 3 months after which the cancer progressed and new lesions appeared. A metastasis recurring in the skin was determined to possess 7,288 genes within copy number amplicons, 385 genes exhibiting increased expression relative to other tumours and 9 new somatic protein coding mutations. The observed mutations and amplifications were found to be consistent with resistance to therapy arising through further activation of RET pathway and nascent activation of the AKT pathway.

\section{Conclusion}

Our results provide evidence for the clinical utility of complete genomic characterization and direct in-vivo genome-wide characterization of the mutations accruing within a tumor under drug selection.

\section{Author details}

'Genome Sciences Centre, British Columbia Cancer Agency, Vancouver BC, Canada. ${ }^{2}$ British Columbia Cancer Agency, Vancouver BC, Canada.

${ }^{3}$ Vancouver General Hospital, Vancouver BC, Canada. ${ }^{4}$ Centre for Translational and Applied Genomics of British Columbia Cancer Agency and the Provincial Health Services Authority Laboratories, Vancouver, BC, Canada. ${ }^{5}$ Department of Molecular Oncology, BC Cancer Research Centre, Vancouver, BC, Canada.

Published: 23 July 2010

doi:10.1186/1471-2105-11-S4-O3

Cite this article as: Jones et al:: Genomic analysis of a rare human tumor. BMC Bioinformatics 2010 11(Suppl 4):O3.

*Correspondence: sjones@bcgsc.ca

'Genome Sciences Centre, British Columbia Cancer Agency, Vancouver BC, Canada 